\title{
The irreversible part of special relativity
}

\author{
Kuyukov Vitaliy Petrovich \\ vitalik.kayukov@mail.ru
}

SFU, Russia

In this paper we consider the existence of a general contribution of the entropy of entanglement on the geometry of Minkowsky space-time. The basic premise is the limit of the entropy of the Hawking of Bekenstein.

Thermodynamics of black holes explicitly, it turns out that the event horizon has a maximum amount of entropy

$$
\begin{gathered}
S_{\max }=\frac{c^{3} A}{4 G h} \\
l_{p}^{2}=\frac{G h}{c^{3}}
\end{gathered}
$$

In addition, any surface of the light sphere is the ultimate limit of the growth of entropy.

But how to be with static surfaces or surfaces with slow speeds?

For a start, consider the motion of some frames (flat screen) at a speed below the speed of light.

$$
\vec{v}=\frac{d \vec{r}}{d t}
$$

Let the screen surface is perpendicular to the vector of its speed.

Then make a necessary and sufficient definition, the entropy of entanglement of flat screen is proportional to the product of the velocity to the square of the screen.

$$
S(A)=\frac{c^{2}}{4 G h} \vec{v} \vec{A}
$$

This definition satisfies the general constraint on the entropy of the screens.

In addition, this equation gives a qualitative interpretation of the theorem of addition of velocities in special relativity based on the summary of entropy.

Consider the motion of a flat screen along selected axis

$$
\begin{gathered}
S\left(A_{y z}\right)=S_{y z}=\frac{c^{2}}{4 G h} v_{x} A_{y z} \\
A_{y z} \perp x
\end{gathered}
$$

His speed in some reference frame

$$
v_{x}=\frac{4 G h}{c^{2}} \frac{S_{y z}}{A_{y z}}
$$


According to the special relativity addition of velocities of the screen and moving system relative to the frame of rest defined by the formula

$$
v=\frac{v_{1}+v_{2}}{1+\frac{v_{1} v_{2}}{c^{2}}}=4 l_{p}^{2} c \frac{\frac{S_{1}}{F}+\frac{S_{2}}{F}}{1+16 l_{p}^{4} \frac{S_{1}}{F} \frac{S_{2}}{F}}
$$

Now recall the simplest definition of entropy through probability of state

$$
S=-\ln p
$$

In this case, the final speed of the screen in the rest frame in a probabilistic way the entropy will be

$$
\begin{gathered}
v=-\frac{4 G h \ln p}{c^{2}} \frac{\ln }{F}=-4 l_{p}^{2} c \frac{\frac{\ln p_{1}}{F}+\frac{\ln p_{2}}{F}}{1+16 l_{p}^{4} \frac{\ln p_{1}}{F} \frac{\ln p_{2}}{F}} \\
4 l_{p}^{2} \frac{\ln p}{F}=4 l_{p}^{2} \frac{\frac{\ln p_{1}}{F}+\frac{\ln p_{2}}{F}}{1+16 l_{p}^{4} \frac{\ln p_{1}}{F} \frac{\ln p_{2}}{F}}
\end{gathered}
$$

Now do the conversion probability is given to the local mind

$$
p^{*}=p^{\frac{4 l_{p}^{2}}{F}}
$$

In this case, the sum of the velocities is taken as the summary of the entropies in probabilistic form. In information theory there is a similar formula to calculate the sum of information entropies in general.

$$
\ln p^{*}=\frac{\ln p_{1}^{*}+\ln p_{2}^{*}}{1+\ln p_{1}^{*} \ln p_{2}^{*}}
$$

Thus, the entropy of entanglement of flat boundaries is closely related to the theorem of addition of velocities and the nature of relativity.

\section{Time and growth entropy of entanglement of space.}

According to special relativity the time goes differently in different points in space for reference frames moving with constant speed

$$
\vec{v}=c^{2} \nabla t
$$

This effect is called relativity of simultaneity of events

$$
v_{x}=c^{2} \frac{t_{2}-t_{1}}{x_{2}-x_{1}}
$$

Consider the motion of the small screen size.Let the entropy of the screen, taking into account the relativity of time, be determined by the formula 


$$
\delta S=\frac{c^{2}}{4 G h} \vec{v} \vec{A}=\frac{c^{4}}{4 G h} \nabla t \delta \vec{A}
$$

To simplify, consider a spherical coordinate system, then the screen area is proportional to the solid angle

$$
\delta A=2 r^{2} \delta \Omega
$$

In this case, the determination of the entropy growth of entanglement and time will be

$$
\begin{aligned}
& \delta S=\frac{c^{4}}{2 G h} \frac{\partial t}{\partial r} r^{2} \delta \Omega \\
& d t=\frac{2 G h}{c^{4}} \frac{\partial S}{r^{2} \partial \Omega} d r
\end{aligned}
$$

A complete determination of the difference in time using these formulas can only be made by taking into account the transition to the consideration of the spatial entropy of space

$$
S(A) \rightarrow S(V)
$$

In this case, for some small region of the volume of space

$$
\delta t d \Omega=\frac{2 G h}{c^{4}} \frac{d S}{r}
$$

We make a definition, in the form of an integral over the entire volume of space and a complete solid angular rotation.

$$
2 \pi t=\int \delta t d \Omega=t \oint d \Omega=\frac{2 G h}{c^{4}} \int \frac{d S}{r}
$$

Hence, the flow of time is directly proportional to the total integral of the growth of entropy of entanglement throughout the infinite volume of space.

$$
t=\frac{G h}{\pi c^{4}} \int_{V \rightarrow \infty} \frac{d S}{r}
$$

This formula connects time and the confusion of space itself.

A more accurate determination of the moment of time relative to the selected point in space will be

$$
\begin{gathered}
t\left(r_{0}\right)=\frac{G h}{\pi c^{4}} \int_{V \rightarrow \infty} \frac{d S}{\left|\vec{r}-\vec{r}_{0}\right|} \\
S=S(V)>0
\end{gathered}
$$

In addition, the interval also includes the entanglement of the space-time geometry.

$$
l^{2}=(c t)^{2}-x^{2}-y^{2}-z^{2}=\left(\frac{l_{p}^{2}}{\pi} \int \frac{d S}{r}\right)^{2}-x^{2}-y^{2}-z^{2}
$$




$$
t=\frac{G h}{\pi c^{4}} \int_{V \rightarrow \infty} \frac{d S}{r}
$$

In this form, the nature of space-time is closely related to the concepts of entropy of entanglement and information. Obviously, the further development of this approach has an important understanding of the nature of relativistic effects, that is, the essence of the real world. 